\title{
The study of internal mammary lymph node dissection guided by modified radiotracer injection technique in breast cancer-a case report and review
}

\author{
Zhao $\mathrm{Bi}^{1,2}$, Peng Chen ${ }^{2}$, Xian-Rang Song ${ }^{2}$, Yong-Sheng Wang ${ }^{2}$ \\ ${ }^{1}$ School of Medicine and Life Sciences, University of Jinan-Shandong Academy of Medical Sciences, Jinan 250200, China; ${ }^{2}$ Shandong Cancer \\ Hospital and Institute, Shandong First Medical University and Shandong Academy of Medical Sciences, Jinan 250117, China \\ Correspondence to: Yongsheng Wang. Shandong Cancer Hospital Affiliated to Shandong University, Ji Yan Road 440, Jinan 250200, China. \\ Email: wangysh2008@aliyun.com.
}

\begin{abstract}
In addition to axillary lymph node (ALN), internal mammary lymph node (IMLN) could also provide important prognostic information. In this paper, we will introduce a case of breast cancer patient whose preoperative lymphoscintigraphy revealed that there were "hot-spots" in bilateral intercostal space. The bilateral IMLN found by preoperative lymphoscintigraphy is a rare phenomenon. She received ipsilateral internal mammary sentinel lymph node biopsy (IM-SLNB) and IMLN dissection and contralateral IM-SLNB. She was diagnosed as pT2N3bM1 breast cancer based on the positive IMLN and positive ALN. After performing surgery, the pathology indicated: (left breast) invasive ductal carcinoma $\left(3.0 \times 3.0 \mathrm{~cm}^{2}\right)$, ALN (3/30), ipsilateral internal mammary sentinel lymph node (IMSLN) (1/2), IMLN (0/2); contralateral IMSLN (1/1). After performing IMLN surgery, the pathology staging increased from pT2N1aM0 to pT2N3bM1. And the irradiation therapy choice had been changed, she received irradiation therapy include chest wall, supraclavicular region, ipsilateral IMLN and contralateral IMLN. The treatment benefit had been increased. When the ipsilateral internal mammary lymphatic vessels were obstructed, deep lymphatic system might drain from ipsilateral IMLN to contralateral IMLN. The contralateral IMLN metastasis belongs to distant metastasis. The IMLN irradiation therapy should be tailored and balanced based on the statues of IMLN. With effective application of systemic therapy, the localized treatment advantage benefited from IMLN surgery might be transferred to survival benefit.
\end{abstract}

Keywords: Breast cancer; internal mammary lymph node (IMLN) dissection; modified injection technique; lymphoscintigraphy; case report

Submitted Nov 17, 2019. Accepted for publication Dec 09, 2019.

doi: 10.21037 /gs.2019.12.19

View this article at: http://dx.doi.org/10.21037/gs.2019.12.19

\section{Introduction}

Internal mammary lymph node (IMLN) and axillary lymph node (ALN) belong to the "first station" lymph node of breast cancer lymphatic drainage, and internal mammary lymphatic vessel is one of the most important metastatic pathways of breast cancer. For breast cancer patients, IMLN metastases has a similar prognostic effect as ALN, which is also important for regional staging and subsequent treatment $(1,2)$. With the appearance of "modified injection technique", the identification rate of internal mammary sentinel lymph node (IM-SLN) has been significantly improved. Internal mammary-sentinel lymph node biopsy (IM-SLNB) might become a minimally invasive technique to evaluate the status of IMLN effectively as well as to guide the individualized diagnosis/treatment (3). To validate the accuracy of IM-SLNB, our center started a multi-center validation trial to perform IMLN dissection on patients with positive ALNs when they finished IMSLNB (Clinical Trial: NCT03024463). The bilateral IMLN found by preoperative lymphoscintigraphy is a rare 


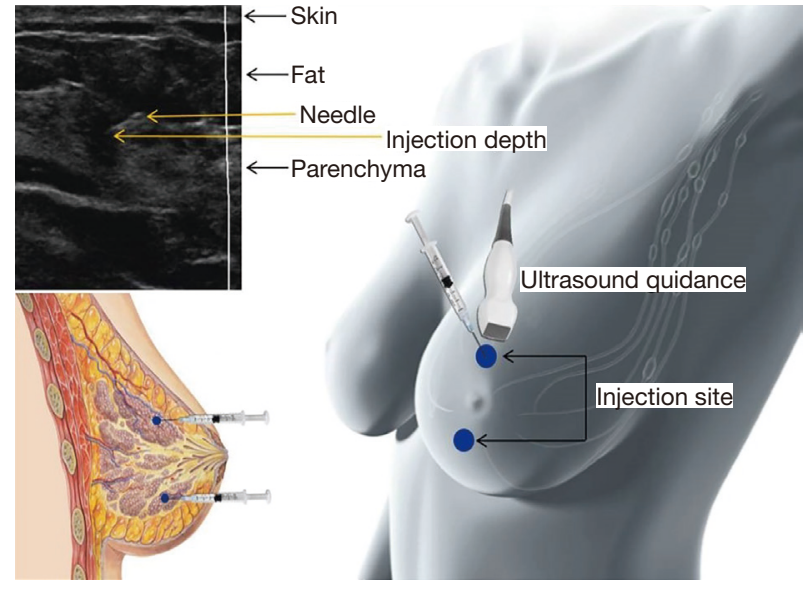

Figure $1{ }^{99} \mathrm{~m}$ Tc-labeled sulfur colloid was injected into the mammary gland at 6 and 12 o'clock of the areola surrounding area guided by modified radiotracer injection technique.

phenomenon. Here we will introduce a case that one female patient treated in our hospital in January 2019. Preoperative lymphoscintigraphy revealed that there were "hot-spots" in left and right second intercostal space. So, she received ipsilateral IM-SLNB and IMLN dissection. At the same time, contralateral IM-SLNB guided by modified injection technique was also performed. Here is the introduction of the case. We present the following case in accordance with the CARE Guideline.

\section{Case presentation}

Our case was a 61-year-old woman with a painless tumor about $3.5 \times 3.0 \mathrm{~cm}^{2}$ in her left breast for one week. She was admitted to our hospital in January, 2019. Pathology result of preoperative core needle biopsy confirmed invasive ductal carcinoma. Ultrasonography revealed several enlarged ALNs, and ultrasound-guided fine needle aspiration (FNA) was performed on the largest one $\left(1.5 \times 1.0 \mathrm{~cm}^{2}\right)$ with malignant cytology discovery. Magnetic resonance imaging revealed that an enlarged IMLN $\left(0.7 \times 0.7 \mathrm{~cm}^{2}\right)$ was located in left second intercostal space. Due to the IMLN had deep anatomical position, the FNA biopsy could not reach the proper position. Imaging examination found no metastases in bone, brain or liver. The clinical stage for this patient was cT2N2M0, stage IIIA. This patient had no relevant family, psycho-social, and genetic history. The study was approved by the Shandong Cancer Hospital Affiliated to Shandong University Ethics Committee. Informed consent was obtained from this patient for the publication of this case report.

She received surgery in 10th January, 2019. Under the guidance of ultrasound, thirty-seven $\mathrm{MBq}$ of ${ }^{99 \mathrm{~m}} \mathrm{Tc}$-labeled sulfur colloid ( $\left.{ }^{99 \mathrm{~m}} \mathrm{Tc}-\mathrm{SC}\right)(1.2 \mathrm{~mL}$ volume) was injected into the left mammary gland at 6 and 12 o'clock of the areola surrounding area guided by modified radiotracer injection technique $15 \mathrm{~h}$ before surgery (Figure 1). Preoperative lymphoscintigraphy revealed that there was a "hot-spot" in left second intercostal space. At the same time, right second intercostal space also had a "hot-spot" scanned by lymphoscintigraphy (Figures 2,3). Blue dye $(4 \mathrm{~mL})$ was injected subcutaneously around the tumor $10 \mathrm{~min}$ before surgery. Then the patient received mastectomy and axillary lymph node dissection (ALND). After finishing breast and axillary surgery, the first IM-SLN was found by the handheld gamma probe in the left second intercostal space as the lymphoscintigraphy revealed. Using the mastectomy incision, from the position where IM-SLN was located, the pectoral major muscle fibers were separated to expose the posterior intercostal space. The external and internal intercostal muscles were divided from the sternal border. Then the first IM-SLN was removed (Figure 4). In the left third intercostal space, we found another IM-SLN using gamma probe. This IM-SLN was removed in the same way. The first IM-SLN was about $0.7 \mathrm{~cm}$ in diameter and nuclide count was 10 , it did contain metastases after routine pathology. The second IMSLN was about $0.5 \mathrm{~cm}$ in diameter, and the nuclide count was 2, it was negative in the end. After finished IM-SLNB, the IMLN dissection was performed. The pectoralis major muscle was severed at the edge of sternal border from first rib to the fourth rib. Then resection of the intercostal muscles and careful opening of the thin anterior leaflet of the parietal pleura in the parasternal area. Ligation the internal mammary blood vessel at the first and third intercostal. Cutting the second and third costal cartilages in their sternal junctions using a scalpel. Ribs traction toward the lateral side of the chest to allow IMLN dissections. Excision of any lymph node tissues found, including surrounded fatty tissues from first to the third intercostal. Then the costal cartilages were sutured (Figure 5). The IMLN dissection procedure lasted 40 minutes. After finishing the left IMLN dissection, the right IM-SLNB was performed using a new incision. From the position where IM-SLN was located, skin and subcutaneous tissues were cutting. Using the same method, the right IM-SLNB was performed. In the right second intercostal space, we found the IMSLN of about $0.5 \mathrm{~cm}$ in diameter, and the nuclide count was 25 . The patient had no 



Figure 2 Right and left second intercostal space had "hot-spots" scanned by preoperative lymphoscintigraphy. A, contralateral IMLN, B, ipsilateral IMLN; IMLN, internal mammary lymph node.

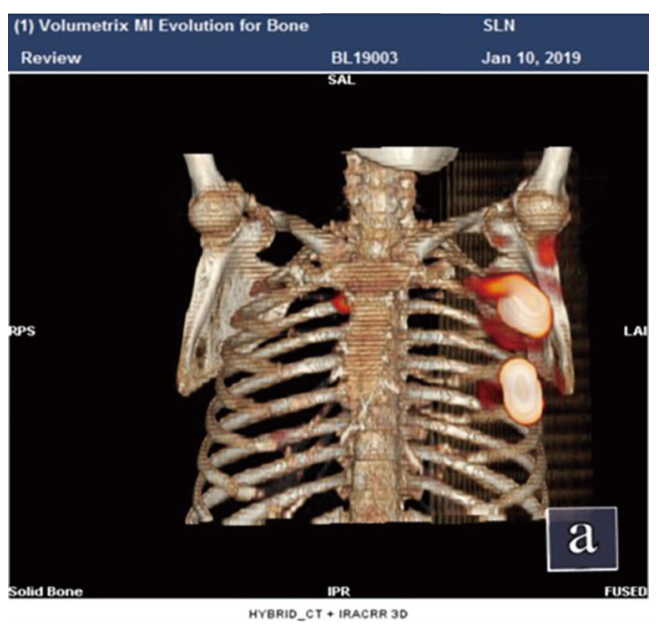

Figure 3 Emission computed tomography image before surgery.

complications.

The routine pathology indicated: (left breast) invasive ductal carcinoma $\left(3.0 \times 3.0 \times 2.0 \mathrm{~cm}^{3}\right)$, grade III, $\operatorname{ALN}(3 / 30)$; (left) IM-SLN (1/2), IMLN (0/2); (right) IM-SLN (1/1). The immune-histochemical finding presented: ER (-), PR $(-)$ and HER-2 negative, the Ki-67 labeling index was about $20 \%$. Since the contralateral IMLN metastasis belongs to

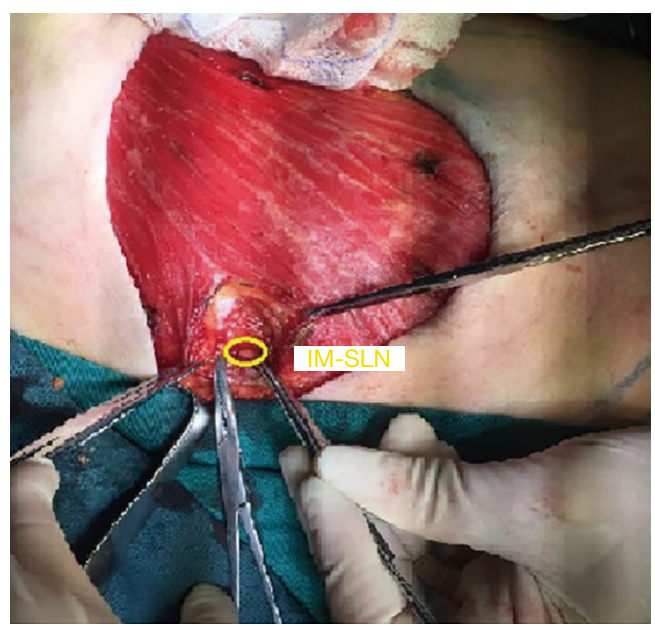

Figure 4 The IM-SLN was removed by IM-SLNB. IM-SLN, internal mammary sentinel lymph node; IM-SLNB, internal mammary sentinel lymph node biopsy.

distant metastasis, the patient's pathological stage from pT2N3bM0, stage IIIC become pT2N3bM1, stage IV. According to the 2019 breast National Comprehensive Cancer Network (NCCN) guidelines, this patient received standard AC (Pharmorubicin $100 \mathrm{mg} / \mathrm{m}^{2} \mathrm{~d} 1$ q21d + Cyclophosphamide $\left.600 \mathrm{mg} / \mathrm{m}^{2} \mathrm{~d} 1 \mathrm{q} 21 \mathrm{~d}\right) \times 4$ times followed by P (Paclitaxel $\left.175 \mathrm{mg} / \mathrm{m}^{2} \mathrm{~d} 1 \mathrm{q} 14 \mathrm{~d}\right) \times 4$ times 

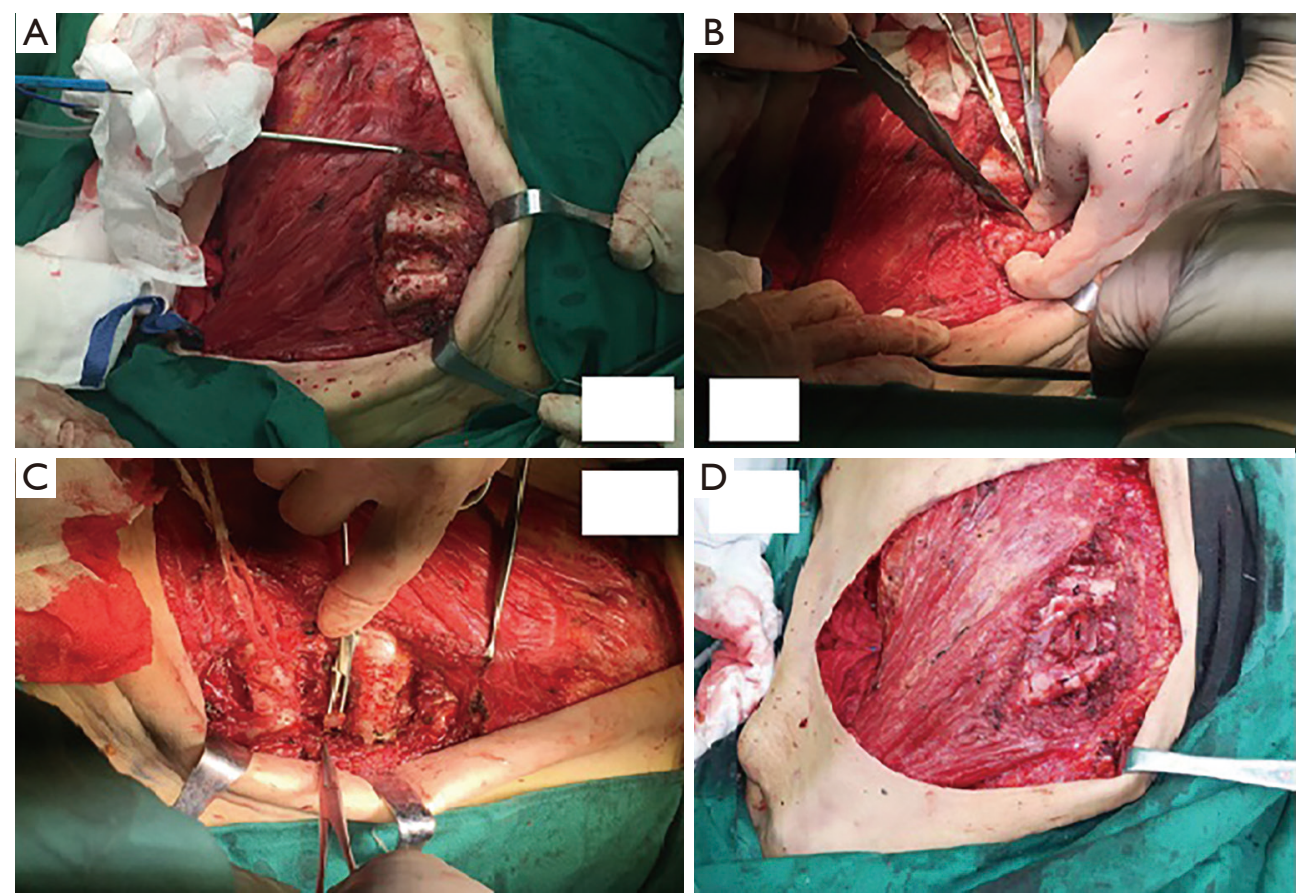

Figure 5 The procedure of IMLN dissection. (A) The pectoralis major muscle was severed from first rib to the fourth rib; (B) cutting the second and third costal cartilages; (C) excision of lymph node tissues from 1st to the 3rd intercostal; (D) suture of costal cartilages. IMLN, internal mammary lymph node.

for chemotherapy. The treatment benefit of this patient had been increased. In the choice of irradiation, she finally received irradiation therapy include ipsilateral chest wall, supraclavicular region and ipsilateral IMLN. At the same time, as to the pathological stage changed, the contralateral IMLN irradiation therapy was also performed.

\section{Discussion}

The IMLN and ALN belong to the "first station" lymph node of breast cancer lymphatic drainage, which is one of the most important metastatic pathways of breast cancer (1). The metastasis rate of IMLN is $18-33 \%$. For patients with positive ALNs, an estimated $28-52 \%$ of them might also have IMLN metastases, while $2-11 \%$ patients with negative ALNs have IMLN involvement (4). The retrospective study of Huang et al. (5) showed that although there was no important difference in tumor size among IMLN metastasis ( $\mathrm{P}>0.01)$, significant difference between IMLN metastasis and ALN metastasis, age, and tumor location (both $\mathrm{P}<0.05$ ) were observed. Coombs et al. (6) reported that patients with lymphatic vascular invasion had high risk to present with IMLN metastasis $(\mathrm{P}=0.032)$. The lymphatic drainage pathway study of Suami et al. (7) showed that the internal mammary lymphatic vessels were identified alongside the internal mammary blood vessels, deep to the parietal pleura, with IMLN present in the intercostal spaces. Collecting lymphatics passed through the intercostal muscle beside each perforating blood vessel to join the internal mammary lymphatic system. Moreover, no apparent connection was observed between the collecting lymphatics accompanying the branches of the internal mammary blood vessels and the superficial collecting lymphatics. However, the ipsilateral internal mammary lymphatic group could connect with the contralateral group. When the ipsilateral internal mammary lymphatic vessels were obstructed, deep lymphatic system might drain to contralateral IMLN. This connection of the internal mammary lymphatic groups might be responsible for contralateral IMLN metastasis.

According to the American Joint Committee on Cancer eighth edition manual, the regional lymph node metastasis includes ipsilateral ALN, supraclavicular, subclavicular and IMLN (1). The contralateral IMLN does not belong to regional lymph nodes. The contralateral IMLN metastasis belongs to distant metastasis. Unfortunately, this patient had contralateral IMLN metastasis, the patient's 
pathological stage become pT2N3bM1 after she received ipsilateral IMLN dissection and contralateral IM-SLNB. The treatment and prognosis of this patient have changed.

With the continuous maturation of radiotherapy technology, IMLN radiotherapy has gained more and more attention due to the impact on survival improvement. The meta-analysis of three clinical trials (EORTC, MA.20, and French trials) confirmed that the 10-year overall survival (OS) and disease-free survival could be significantly improved when there were additional IMLN and supraclavicular region radiotherapy (8). The 2019 NCCN Guidelines recommended IMLN radiotherapy at risk in patients with $\geq 4$ positive ALNs (category 1)/strongly suggest IMNI in patients with 1 to 3 positive ALNs (category 2A) (9). At present, the indication of IMLN radiotherapy mainly depends on the high-risk factors of IMLN metastases (only patients with high-risk metastasis factors were selected without IMLN pathology assessment). However, patients with high-risk factors of IMLN metastases do not necessarily have IMLN metastases, and low-risk patients could not be excluded of the possibility of IMLN metastases. The treatment strategy dependent on risk factors could lead to a certain degree of under-/overtreatment. Taking the pulmonary and cardiac toxicities of IMLN radiotherapy into account, the pathological status of IMLN is superior to the high-risk factors for the guidance of IMLN radiotherapy. In our case, this patient received radiotherapy in ipsilateral chest wall, supraclavicular region and IMLN. Due to the contralateral IMLN metastasis, this patient also received contralateral IMLN radiotherapy.

IMLN has been well known to usually have a deep anatomical position and small diameter $(<0.5 \mathrm{~cm})$, and the sensitivity of imaging examination cannot meet the clinical requirements to detect IMLN metastases. With the development of SLNB technique, IM-SLNB is expected to be a minimally invasive diagnostic technique in assessing the status of IMLN metastasis (2). The accuracy of axillarySLNB (A-SLNB) has been proved by ALND. The lymphatic vessels drainage from whole breast parenchyma could reach to the same axillary sentinel lymph node (A-SLN). According to the A-SLN lymphatic drainage pattern, Cong et al. (10) hypothesized that IMSLN receives lymphatic drainage from not only the primary tumor area, but also the entire breast parenchyma. To observe the accuracy of IM-SLNB, different tracers (radiotracer and fluorescence tracer) were injected into different locations of the breast (the primary tumor and subareolar plexus). The results showed the lymphatic drainage from different location of the breast could reach the same IMLN. At the

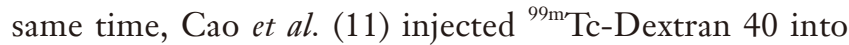
breast intra-parenchyma by modified radiotracer injection technique. Subsequently, SPECT/CT combination images were performed to identify the radioactive lymph vessels and IMLNs. The result showed that drainage lymphatic vessels were found from injection point to the first IMLN (IM-SLN) after $10.5 \pm 0.35$ min radiotracer injection. But there was no anatomical study about IMLN lymphatic drainage due to the abandon of extended radical mastectomy.

Following early reports of worse overall prognosis in patients with IMLN metastasis, a multicenter randomized trial comparing extended radical mastectomy to the modified radical mastectomy was initiated. The results of this trial demonstrated that extended mastectomy did not improve OS (12). Subsequent updates confirmed no survival improvement with extended mastectomy. Veronesi et al. (13) also showed that extended mastectomy had no survival advantage over modified radical mastectomy after 30 years of follow-up. So, the extended radical mastectomy had been abandoned because it might not improve the OS. However, after 20-year of follow-up in Chinese patients, Shen et al. (14) found there was no significantly difference of the OS in stage I patients between the modified radical mastectomy and extended radical mastectomy. While compared with the modified radical mastectomy performed in the same period, stage II-III patients performed with extended radical mastectomy had a significant improvement in the OS (both $\mathrm{P}<0.01$ ). It is worth noting that in foreign countries, because the extended mastectomy started early, systemic adjuvant therapy has not been implemented in patients who received extended mastectomy. Most patients had distant metastasis in their early treatment (the OS was shorter). But due to the extended mastectomy started later in China, patients who performed extended mastectomy had received systemic adjuvant therapy. The localized treatment advantage benefited from extended mastectomy could be transferred to survival benefit. In the era of molecular typing, with the widespread application of systemic therapy, the significance of IMLN treatment is worth rethinking.

Although the IM-SLNB could guide IMLN radiotherapy, unfortunately, the indication of IM-SLNB had not been confirmed. Recently, our investigators proposed that if IM-SLN is the only metastatic lymph node in IMLN chain, the radiotherapy and its associated complications could be avoided in those patients without positive IM-SLN. Furthermore, after IM-SLNB, if there present metastatic non-SLN (NSLN) in IMLN chain, it 
is important to predict the risk of IM-NSLN metastasis in IM-SLN positive patients. As there is currently no such model, a predictive model for IM-SLN positive patients to avoid radiotherapy is needed in this situation. Therefore, a prospective multi-center study was designed as a clinical verification of IM-SLNB (Clinical Trial: NCT03024463). In our current study, all the participants would have the preoperative pathology of invasive breast cancer and positive FNA result in their ALN. ${ }^{99 \mathrm{~m}} \mathrm{Tc}-\mathrm{SC}$ was injected into the parenchyma using modified radiotracer injection technique 3 18 hours before surgery. IM-SLNB was performed in all participants with IM-SLN visualized on preoperative lymphoscintigraphy and/or detected by the intra-operative gamma probe. All hotspots in the IMLN were harvested and intra-operative identification of the IM-SLN was based on gamma probe detection. IMLN dissection of the first to the third intercostal was performed immediately after IM-SLNB. Finally, all the IMLN dissected were assessed post-operatively by H\&E and Cytokeratin-19 stained immunohistochemistry. Up to now, a total of 73 IM-SLN of 61 patients were obtained. The false-negative rate of our study was $3.85 \%$. The relevant research results will be announced in the next year.

In conclusion, the IMLN have played a very important role in the surgical approach of breast cancer. When the ipsilateral internal mammary lymphatic vessels are obstructed, deep lymphatic system might drain from ipsilateral IMLN to contralateral IMLN. IM-SLNB guided by the modified radiotracer injection technique has a high accuracy. We recommend IM-SLNB should be performed in all patients, especially in patients with clinically positive ALN. IMLN radiotherapy should be tailored and balanced based on the statues of IM-SLN.

\section{Acknowledgments}

Funding: This work was supported by National Natural Science Foundation of China (81672638) and Shandong Provincial Key Research and Development Program (2017CXGC1207, 2019GSF108179).

\section{Footnote}

Conflicts of Interest: All authors have completed the ICMJE uniform disclosure form (available at http://dx.doi. org/10.21037/gs.2019.12.19). The authors have no conflicts of interest to declare.
Ethical Statement: The authors are accountable for all aspects of the work in ensuring that questions related to the accuracy or integrity of any part of the work are appropriately investigated and resolved. Informed consent for the publication of the report, and the accompanying images, was provided by the patient. The submission version of the report was read by the patient, and the report's content was confirmed as being correct to the best of her knowledge.

Open Access Statement: This is an Open Access article distributed in accordance with the Creative Commons Attribution-NonCommercial-NoDerivs 4.0 International License (CC BY-NC-ND 4.0), which permits the noncommercial replication and distribution of the article with the strict proviso that no changes or edits are made and the original work is properly cited (including links to both the formal publication through the relevant DOI and the license). See: https://creativecommons.org/licenses/by-nc-nd/4.0/.

\section{References}

1. Giuliano AE, Connolly JL, Edge SB, et al. Breast CancerMajor changes in the American Joint Committee on Cancer eighth edition cancer staging manual. CA Cancer J Clin 2017;67:290-303.

2. Bi Z, Qiu PF, Wang YS. Advances in diagnosis and treatment of internal mammary lymph node of breast cancer. Chin J Clin Oncol 2017;44:1104-7.

3. Qiu PF, Cong BB, Wang YS, et al. Internal mammary sentinel lymph node biopsy with modified injection technique: high visualization rate and accurate staging. Medicine (Baltimore) 2015;94:e1790.

4. van der Ent FW, Kengen RA, van der Pol HA, et al. Halsted revisited: internal mammary sentinel lymph node biopsy in breast cancer. Ann Surg 2001;234:79-84.

5. Huang O, Wang L, Shen K, et al. Breast cancer subpopulation with high risk of internal mammary lymph nodes metastasis: analysis of 2,269 Chinese breast cancer patients treated with extended radical mastectomy. Breast Cancer Res Treat 2008;107:379-87.

6. Coombs NJ, Boyages J, French JR, et al. Internal mammary sentinel nodes: ignore, irradiate or operate? Eur J Cancer 2009;45:789-94.

7. Suami H, Pan WR, Mann GB, et al. The lymphatic anatomy of the breast and its implications for sentinel lymph node biopsy: a human cadaver study. Ann Surg Oncol 2008;15:863-71. 
8. Budach W, Kammers K, Boelke E, et al. Adjuvant radiotherapy of regional lymph nodes in breast cancer a meta-analysis of randomized trials. Radiat Oncol 2013;8:267-73.

9. Grasishar WJ, Anderson BO, Balassanian R, et al. Breast cancer, Version 1.2019 featured updates to the NCCN guidelines [EB]. National Comprehensive Cancer Network. Available online: http://www.NCCN.org

10. Cong BB, Qiu PF, Liu YB, et al. Validation study for the hypothesis of internal mammary sentinel lymph node lymphatic drainage in breast cancer. Oncotarget 2016;7:41996-2006.

11. Cao XS, Yang GR, Wang YS, et al. The Lymphatic

Cite this article as: Bi Z, Chen P, Song XR, Wang YS. The study of internal mammary lymph node dissection guided by modified radiotracer injection technique in breast cancer-a case report and review. Gland Surg 2020;9(2):430-436. doi: 10.21037/ gs.2019.12.19
Drainage Pattern of Internal Mammary Sentinel Lymph Node Identified by Small Particle Radiotracer (99mTcDextran 40) in Breast. Cancer Res Treat 2019;51:483-92.

12. Lacour J, Bucalossi P, Cacers E, et al. Radical mastectomy versus radical mastectomy plus internal mammary dissection. Five-year results of an international cooperative study. Cancer 1976;37:206-14.

13. Veronesi U, Marubini E, Mariani L, et al. The dissection of internal mammary nodes does not improve the survival of breast cancer patients. 30-year results of a randomized trial. Eur J Cancer 1999;35:1320-5.

14. Shen ZZ, Han QX, Li YR. Extended radical mastectomy: an analysis of 1091 cases. Shanghai Med 1982;5:499-503. 